\title{
Maïssa Bey, Pierre Sang Papier ou Cendre
}

\section{Carmelina Imbroscio}

\section{(2) OpenEdition}

\section{Journals}

\section{Edizione digitale}

URL: http://journals.openedition.org/studifrancesi/6461

DOI: 10.4000/studifrancesi.6461

ISSN: 2421-5856

\section{Editore}

Rosenberg \& Sellier

\section{Edizione cartacea}

Data di pubblicazione: 1 novembre 2010

Paginazione: 599-600

ISSN: 0039-2944

\section{Notizia bibliografica digitale}

Carmelina Imbroscio, «Maïssa Bey, Pierre Sang Papier ou Cendre», Studi Francesi [Online], 162 (LIV | III) | 2010, online dal 30 novembre 2015, consultato il 12 janvier 2021. URL: http://

journals.openedition.org/studifrancesi/6461 ; DOI: https://doi.org/10.4000/studifrancesi.6461

Questo documento è stato generato automaticamente il 12 janvier 2021.

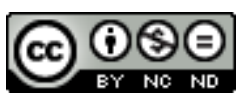

Studi Francesi è distribuita con Licenza Creative Commons Attribuzione - Non commerciale - Non opere derivate 4.0 Internazionale. 


\title{
Maïssa Bey, Pierre Sang Papier ou Cendre
}

\author{
Carmelina Imbroscio
}

\section{NOTIZIA}

MAÏSSA BEY, Pierre Sang Papier ou Cendre, La Tour d'Aigues, Éditions de l'Aube, 2008, pp. 206.

1 Maïssa Bey può essere definita scrittrice dell'esilio anche se risiede in Algeria, sua terra natale. È possibile farlo se utilizziamo per lei la categoria dell'esilio morale, culturale, e se riconosciamo nell'esiliato (e in particolare nell'esiliata, visto che il legame col territorio è più declinabile al femminile) l'individuo preso tra due culture, quella di provenienza e quella di incontro, in una combinazione spesso conflittuale e confusa tra i valori di una tradizione che può rivelarsi oppressiva, ma che è innegabilmente costitutiva, e i valori di una nuova cultura che si vorrebbe abbracciare senza negarsi, ma che talvolta respinge ai margini chi non abiura.

2 Pierre Sang Papier ou Cendre, che ha vinto «le Prix du Roman» al Salone Internazionale del Libro di Algeri (2008), è uno scritto intriso di esperienza autobiografica - come ci comunica la stessa scrittrice nei riferimenti al vissuto personale che sempre ritornano nelle sue pagine - adattato anche per il teatro nel febbraio 2008 (messa in scena di JeanMarie Lejude), col titolo di Madame Lafrance; e di fatto è proprio "Madame Lafrance" l'onnipresente protagonista del libro, inquietante allegoria dai mille volti, che per 132 anni si è installata da padrona di casa in terra d'Algeria. Pierre Sang Papier ou Cendre ne percorre la parabola storica: ora seducente chimera, vessillifera di valori di "civiltà", benigna e paziente dispensatrice di lumi e di idee di fratellanza, poi benefattrice offesa dall'ingratitudine dei protetti e grande madre vendicativa, infine interlocutrice smarrita, quando - di fronte alla determinata azione del FNL - gli stessi "francesi di Francia" si dividono sul merito dell'istanza di indipendenza degli algerini. Persa la baldanza, «Madame Lafrance est divisée. Madame Lafrance est fébrile [...] Madame 
Lafrance est ébranlée» (pp. 179 e 187). Ci si fronteggia, da entrambe le parti, sotto la sua stessa bandiera: il sogno coloniale ha mostrato la corda, e la generosa bonomìa che accordava ai rozzi indigeni la possibilità di accedere al privilegio dell'identità francese ha rivelato la sua ideologia di dominio. Eppure Maïssa Bey affida l'evocazione della libertà al patrimonio delle lettere di Francia: il titolo del libro rimanda a Éluard, l'epigrafe in esergo è tratta dalla Marsigliese, Camus è personaggio che anima alcune pagine interne e frammenti sparsi rendono omaggio a vari scrittori francesi, elencati poi nelle pagine finali...

Il fatto è che la Francia rappresenta per Maïssa Bey «l'une et l'autre» (è questo il titolo di un suo recente saggio, L'Aube, 2009): è l'occupante che ha dominato, mortificato e ucciso (anche il padre della scrittrice, resistente, fellaga, è stato torturato e giustiziato), ma è anche una cultura che le è entrata nel sangue, una lingua che le appartiene, che ama, e nella quale esprime la sua visione del mondo; "l'une et l'autre" è anche la sua Algeria: cultura delle origini, matrice identitaria, ma anche spazio claustrante, rappresentante di valori immutabili, dei quali l'Islam fa depositaria la donna. "L'une et l'autre" sono anche le due culture che si fronteggiano: quella delle radici e quella dell'emancipazione dalle stesse, entrambe affascinanti, entrambe insidiose, entrambe portatrici di spazi aperti e di barriere. Ne derivano vacillamenti identitari che fanno sentire «à la lisière de deux mondes» (L'une et l'autre, cit., p. 40). Questa identità lacerata si era mostrata ancor più ferita in Entendez-vous dans les montagnes... (L'Aube, 2002), racconto che vede la protagonista, le cui vicende biografiche sono molto prossime a quelle della scrittrice, viaggiare in Francia - esule dal suo paese sconvolto da una nuova barbarie di sangue - seduta nello stesso scompartimento di quello che, nel dialogo faticosamente avviato, si rivelerà essere stato proprio uno dei torturatori del padre. La vicenda che si svolge nel treno si complica di quella narrata nel libro che la protagonista legge, Le Liseur (Der Vorleser, di Bernhard Schlink, 1995), che evoca gli eccidi nazisti e induce a riflessioni sulla banalità del male... il torturatore, di cui ha immaginato per anni fisionomia, identità, può quindi essere quel compìto, imbarazzato e tormentato anziano che le siede di fronte... Dove ritrovarsi? Intorno a cosa costruirsi un io identitario se il proprio paese, a così duro prezzo difeso, è quello in cui si compiono ora gli orrori da cui fugge e se la Francia che l'accoglie è anche quella che ha represso le istanze di libertà e armato la mano di chi ha ucciso, tra gli altri, suo padre? Eppure, suggerisce Maïssa Bey nel suo percorso di riflessione affidata alla scrittura, l'identità "scomposta" può divenire identità molteplice, nella lisière sta la capacità di vedere in più campi, di mettersi in relazione dinamica con l'altro senza negarsi. Il finale di Entendez-vous [...] sembra aprire uno spiraglio di conciliazione umana tra i due personaggi in scena; L'une et l'autre si chiude con l'invito di Glissant a "vivre une altérité étoilée d'héritages et d'horizons»; Pierre Sang Papier ou Cendre, pur vibrante di sdegno, affida al bambino protagonista la prospettiva di un mondo in cui «finit [...] par s'éteindre l'écho de tous les cris de haine jetés à la face du jour».

L'espatrio permanente, l'esilio continuo, sono il prezzo del rifiuto dell'appartenenza univoca, ma sono anche la garanzia che il percorso non si chiude, che la parola testimoniata, la pagina scritta, restano l'unico, multiforme, di instabile e mutevole ricchezza, patrimonio identitario di chi ha deciso di affidare ad esse il suo percorso di vita. 